\title{
Antioxidant Medications: Facts, Myths and Prospects
}

\section{Abdalla M El-Mowafy*}

Faculty of Pharmaceutical Sciences and Industries, Future University in Egypt, Egypt

\begin{abstract}
This review, while defining antioxidants (AOs), their actions and benefits, more importantly critically highlights and rationally validates many myths and controversies regarding their supplementation. Questions will be answered regarding whether: 1) AOs are such harmless drugs that unlimitedly boost body functions, kill cancer, and blunt aging, 2) AO members are "created equal" so that they are equally effective, 3) AO-fortified food and beverages should be taken as a sole, favorable resource for $\mathrm{AO}$ supplementation, and 4) $\mathrm{AO}$ supplements have always positive impact on athletes' performance and gain such that they can make them increasingly good and fit. Finally, future concerns and directions are discussed.
\end{abstract}

\section{Oxidative Stress (OS)}

Oxidative stress (OS) occurs when cellular endogenous antioxidant machinery gets overwhelmed with reactive oxygen and nitrogen species (RONS) that are simply denoted as "free radicals". RONS can serve some limited physiologic and immunologic functions, as exemplified by nitric-oxide signaling in vascular systems, and by peroxynitriteevoked killing of bacteria in phagocytes. However, RONS are known to mostly trigger detrimental (disruptive or mutagenic) responses. At the molecular level, the latter responses are entailed to either direct disruptive damage of cell components (proteins, lipids, and DNA), or alternatively to modulation of cellular signaling pathways, effectors and transcription factors with ultimate pathologic sequelae. Thus, not surprisingly, OS has evidently been established as a primary antecedent in the etiology, exacerbation and complications of a myriad of disorders that could range from fatigue to stroke, and from impotence to aging. These diseases, likewise, encompass both curable and degenerative diseases such as arrhythmias, hepatitis, arthritis, heart failure, coronary heart disease, diabetes, Parkinsonism, Alzheimer's disease and cancer. Conceivably, therefore, combating OS has been speculated as a worthwhile cause to prevent/cure numerous such devastating diseases.

Facts about Antioxidants (AOs): An Armamentarium of Diverse Members

AOs include "endogenous" enzymes and proteins, such as catalase, superoxide dismutase and glutathione, as well as "exogenous" dietary ones such as vitamins (C, E, A) and polyphenols (catechins and flavonoids) that are found mainly in colored fresh vegetables, berries, and fruits, and in green-tea. AOs scavenge free radicals, or prevent their actions effectively, when used at rather low concentrations. Interestingly, each $\mathrm{AO}$ is specific against one or a few types of radicals/species. Likewise, AOs can either be more effective in lipid medium, as per the cell membrane (like Vit-E, Vit-A, and polyphenols), or be superior in aqueous (watery) medium, as per the cytosol (like Vit-C). Therefore, "antioxidants are not created equal", and thus should accordingly be regarded as an "army" or a "crew" of integrative members, rather than substitutes. Best $\mathrm{AO}$ recipes shall, therefore, comprise a blend of members that belong to different subcategories.

\section{Myths, Controversies and Caveats Regarding AO Actions and Supplementations}

The struggle between AOs and disease has been billed as an epic simulating a battle of "good" against "evil". This concept entailed many controversies, uncertainties and myths. In this vein, many contentions and overstatements on actions of AO have emerged. Examples include
"They conquer cancer!", "They blunt aging!", "They supercharge your immune system!". AO have many such health auras coined to them that were also commercially recruited by food manufacturers and nutrition stores in such a way that mandated serious regulations and arrangements by the FDA.

Therefore, the public remains under the impression that "if AO are supposed to do this much magic for our bodies, who wouldn't desire to consume more of such a helper?" which is indeed a big myth that will be argued against next. Although there is little evidence that high doses of antioxidant supplements may help prevent disease, there are some clues that they may sometimes exacerbate an existing disease. Thus, administration of high doses of beta-carotene or vitamin A, elevates the risk for heart attacks in men and lung cancer in smokers. Likewise, high doses of vitamin-C do not appear to prevent colon cancer, or even enhance life expectancy in people with such type of cancer. Other studies also concluded that large doses of vitamin-E are without effect in lung cancer, heart disease and stroke. Conversely, in experimental animals, Vit-C large doses helped minimize the paraquat-induced carcinogenesis, provided it was given quite a head of paraquat (an insecticide). Conversely, however, when Vit- $\mathrm{C}$ was given several hours after paraquat, it rather triggered a more spread of the cancer. One possible such explanation is that free radicals might still be needed in some concentrations to help kill cancer cells. Even though free radicals have been linked with serious anomalies, like heart disease and cancer, they aren't necessarily nefarious. As said before, immune cells will shoot free radicals onto invading microbes in order to kill them. The ultimate take home message is to stick to rationally prescribed AOs, as deems necessary. Alternatively, people with bad habits, such as smoking and excessive tanning, should count on a dietary AO supplement. Eating at least five servings of diverse fruits and vegetables can provide thousands of $\mathrm{AO}$ to neutralize OS and reap most of health benefits.

That last statement brings in another "myth" that AOs should come only from vegetables and fruits. Indeed, also beans, nuts, seeds,

*Corresponding author: Abdalla M El-Mowafy, Faculty of Pharmaceutical Sciences and Industries, Future University in Egypt, Egypt, Tel: 0020-1009844721; E-mail: aelmowafy@yahoo.com

Received May 16, 2013; Accepted May 17, 2013; Published May 19, 2013

Citation: El-Mowafy AM (2013) Antioxidant Medications: Facts, Myths and Prospects. Biochem Anal Biochem 2: e137. doi:10.4172/2161-1009.1000e137

Copyright: ( 2013 El-Mowafy AM. This is an open-access article distributed under the terms of the Creative Commons Attribution License, which permits unrestricted use, distribution, and reproduction in any medium, provided the original author and source are credited. 
and grains are awash in AOs. Underpinnings of this phenomenon are that all plants produce AOs to fight against predators and UV rays. Furthermore, meat, dairy products, and eggs also contain some AOs, which principally come from the nutrient-loaded plants the animals fed on.

An additional "myth" has been that AO-fortified foods are healthier and can be an adequate supply of our needs of $\mathrm{AO}$ vitamin supplements. Where this can be true in a few cases, it is not always the reality. Therefore, the FDA requires food manufacturers to list the variety of AOs and their amounts in a product. Scrutiny reveals that, in many instances, such beverages provide only a tiny helping of a single AO, like vitamin-E or Vitamin-C. As explained before, because a "variety" of AOs is crucially needed to serve different goals, one would probably not get compensated for his lost ground.

Another controversy has revolved around the impact of $\mathrm{AO}$ supplements on athlete's performance and gain. The type of "myth" here is that (If I exercise while taking supplements, I'll be super-fit). The fact, however, is that working out favors oxidation and increases free radicals, which, paradoxically, was proven to be physiologically good in this particular case. To unravel the mystery, scientists showed that exercise-evoked OS promotes insulin sensitivity and weight loss, thereby limiting the risk of obesity and type- 2 diabetes. Accordingly, weight loss experts declare that taking a large dose of AOs right after a workout could blunt the anticipated benefits of this exercise. Several clues have attested for these envisions. Thus, a German 2009 study showed that when exercisers took the AOs (vit-C and -E), they did not demonstrate the typical post-exercise enhanced insulin sensitivity. In support also were the findings that $\mathrm{AO}$ supplements can even undermine an athlete's power. Thus, a British 2006 study revealed that runners, who received 1.0 gram of vit-C daily for a week, ended up losing most of their muscle strength.

\section{Conclusion and Future Directions}

AO supplementation should be regulated and rationalized to patients. AOs are usually needed in optimal amounts that can be supplied through healthy food intake. In many cases, fruit and vegetable servings can be an adequate supply. When OS is diagnosed, based on a laboratory testing and scrutinized medical checkup, AOs should be prescribed rationally via optimal supplementation recipes. Overdosing with AOs can worsen organ functions and/or exacerbate an existing disease. Athletes, individuals on regular exercise, or those desiring to lose weight should not administer AOs right after their workout, as this can interfere with their body metabolic gain and/or weaken their muscle strength. The prospects and future directions with AOs shall depend not only on our current knowledge with OS and AOs, but also on better future understanding and unveiling of their exact mode of action, molecular triggers, as well as dynamic/kinetic profiles of their individual and combined effects. Implementing most recent, capable, and evidence-based approaches, as those adopted by the OMICs technologies, is one such avenues. Gaining insights into this profound knowledge should avail rational AO prescribing to patients, as merely dictated by their individual extent of suffer and exact needs. 